\title{
Simulation Study on the Effect of Grading Diagnosis and Treatment Based on System Dynamics Model in the Context of Medical Alliance--Taking Jiangsu Province as An Example
}

shaoliang tang ( $\nabla$ tangshaoliang@126.com )

Nanjing University Of Chinese Medicine https://orcid.org/0000-0002-7376-8964

Jiahuan Qiu

Nanjing University of Chinese Medichine

Kean Tang

Tianjing Normal University

Research article

Keywords: medical alliance, Grading diagnosis and treatment, System dynamics model

Posted Date: July 2nd, 2019

DOl: https://doi.org/10.21203/rs.2.10840/v1

License: (c) (i) This work is licensed under a Creative Commons Attribution 4.0 International License.

Read Full License 


\section{Abstract}

Abstract: Objective To study the core influencing factor of the implementation of Grading diagnosis and treatment system in the context of Jiangsu medical alliance. Methods By constructing the grading diagnosis and treatment system dynamics model under the background of medical alliance, the number of first-time treatments at the primary level was taken as the core evaluation index. By intervening on different targets, we would study the influence on the core evaluation index. Results The selected six intervention targets had positive effects on graded diagnosis and treatment. Among them, the intervention of medical resource subsidence, the construction of information platform between medical alliances and the technical level of primary level had the greatest impact. Conclusion In order to better promote the development of the grading diagnosis and treatment system, we can focus on the construction of medical alliances: For example, implementing the sinking of high quality medical resources in the medical alliance to strengthen the discipline construction of primary level; breaking the information island in the medical alliance, and strengthening the construction of information platform; building and expanding the green channel for two-way referral, implementing the differentiation of medical insurance policies, and improving the mechanism of benefit sharing in the medical alliances to gradually realize the orderly rationalization of medical treatment.

\section{Introduction}

\subsection{Promotion of the graded diagnosis and treatment system}

Graded diagnosis and treatment refers to grading the disease according to the Severity, heaviness, urgency and the difficulty of treatment. It requires different levels of medical institutions to undertake the treatments of different grades of diseases, clear the division of labor, and gradually realize reasonable medical treatment ${ }^{[1]}$, which is beneficial to alleviate the problem of "difficult to diagnose, expensive to see a doctor" ${ }^{2}$, , to play the role of medical institutions at all levels in the health system, improve the efficiency of the use of health resources, and promote the formation of a reasonable medical order ${ }^{[3]}$.

In September 2015, the State Council issued the "Guiding Opinions on Promoting the Construction of Graded Diagnosis and Treatment System", clearly proposing an important measure to establish a grading diagnosis and treatment system, and subsequently issued the "13th Five-Year Plan for Deepening the Medical and Health System Reform Plan" issued in 2017. It is proposed that we should insist residents be voluntarily, first treatment in primary medical institutions, policy guidance, innovative mechanisms, and encourage all areas to implement various forms of grading diagnosis and treatment. As the first batch of pilot provinces for comprehensive medical reform, Jiangsu Province continued to promote the optimization of the graded diagnosis and treatment system during this period, and always regarded the establishment and improvement of graded diagnosis and treatment as one of the key tasks of the current medical and health system reform in Jiangsu Province ${ }^{[4]}$. Up to now, the framework of the graded 


\subsection{Implementation of grading diagnosis and treatment in the context of medical alliances}

The medical consortium (simply called the medical alliances) refers to the medical community formed by the vertical or horizontal integration of the resources of different levels and different types of medical institutions in the region, in the form of collaborations such as medical unions or medical groups. The medical alliances is an important step and institutional innovation to deepen the medical reform, and is also an important measure to accelerate the promotion of the graded diagnosis and treatment system.

In October 2018, the Jiangsu Provincial Health and Health Commission issued the "Notice on Accelerating the Pilot Work of the Construction of Closed medical alliances", which clearly defined that the key points are implementing the functional orientation of medical institutions, improving the ability of primary medical institutions services, and realizing two-way referrals, then further improve the management model of the close medical alliances, so as to better implement the grading diagnosis and treatment and meet the health needs of the people, and raise the construction of the medical alliances to a new height, its positive significance does not need to be rumored.

Therefore, during the process of vigorously developing the construction of medical alliances in Jiangsu Province, this study realized the simulation prediction of the graded diagnosis and treatment system by constructing the dynamic model of the graded diagnosis and treatment system in the context of the medical alliances of Jiangsu Province, and explored the core factors of the implementation of the classification diagnosis and treatment system, so that to provide feasible suggestions for further improving the graded diagnosis and treatment system.

\section{Sources And Methods}

\subsection{Source of information}

Through collecting and analyzing essays related to the influencing factors about the graded diagnosis and treatment, medical alliances, and the implementation of the graded diagnosis and treatment system from Chinese Journal Full-text Database (CNKI) and related policy documents of Jiangsu provincial to determine the core influencing factors. Combining the relevant indicators of Jiangsu Statistical Yearbook to make comprehensive analysis.

\subsection{Method}


Evidence-based analysis is one of the important methods to achieve effective decision-making, and evidence-based research based on model simulation can make a forward-looking analysis of policy effects. At present, many countries have applied high-quality model simulation research evidence to health policy and obtained good health output and social performance ${ }^{[5-6]}$. Systemic Dynamics (SD) is a system simulation method proposed by Professor JW Forrester in the 1950s for analyzing enterprise problems such as production management and inventory management ${ }^{[7]}$, originally called industrial dynamics, is a method system for analyzing the evolution of complex systems and information feedback. The basic principle of constructing the SD model is to study the feedback system by establishing the flow and flow rate system, and define the complex system as a high-order, multi-loop and nonlinear feedback structure, and assume the motion of all systems in the world as fluid motion. The use of causality diagrams and system flow diagrams to represent the structure of the system ${ }^{[8]}$ has unique advantages in dealing with long-term, high-order, and multiple feedback relationships, and has been well applied in health system assessment in recent years ${ }^{[9]}$.

The graded diagnosis and treatment system is a complex social system in which the demand side, the supplier, the third party, and various factors such as policy, population development, and economic development work together ${ }^{[10]}$. Due to relationships such as the interaction, reflecting lag, and the multilinear feedback between the internal subsystems and the external environment, the evolution of the system behavior is more complex, and the system dynamics model can be well displayed in the system. The external relationship and its evolution process make the research process more intuitive and easy to explore in the next step. Therefore, this study will use the Vensim PLE32 software to determine the grading diagnosis and treatment system according to the actual operation of the grading diagnosis and treatment system in Jiangsu Province, draw a causal relationship diagram and flow diagram, and then construct a system dynamics model, and select appropriate intervention targets to explore factors that influence the effectiveness of graded care implementation.

\section{Construction Steps Of System Dynamics Model <}

There are six main steps in SD modeling. As shown in Figure 1. First, it is necessary to analyze the important factors affecting the operation of the grading diagnosis and treatment system, and explore the relationship between these factors to establish the causal relationship diagram of the SD model. Secondly, determine the variables and construct the system flow graph, establish the functional relationship between the variables, then perform the authenticity test. Finally, simulate the system to make further research ${ }^{[11]}$.

Figure 1 Grading diagnosis and treatment system dynamics modeling steps

\section{Construction Of Dynamic Model Of Grading Diagnosis And Treatment System Under The Background Of Medical Alliances}




\subsection{Determination of model boundaries}

The grading diagnosis and treatment system is defined as two subsystems: the hospital subsystem and the primary medical institutions subsystem, and the two subsystems realize the integration of system functions through two-way referral ${ }^{[12]}$. The implementation of grading diagnosis and treatment in Jiangsu Province is influenced by the internal environment and the external environment. The internal environment includes two subsystems: supplier and demander. The external environment includes factors such as policy, economy and population, and each factor contains many elements. Together with the grading diagnosis and treatment system, it restricts the implementation of grading diagnosis and treatment from various aspects ${ }^{[13]}$. Based on the induction and analysis of the essays, this study determined that the first diagnosis at the primary medical institutions is the core element of the dynamic model of the grading diagnosis and treatment system . Other elements are included as shown below:

(1) Influencing factors of the demand side: the patient's medical concept, income level, accessibility of medical institutions, medical insurance differentiation level, patient's willingness to grade, etc.

(2) Influencing factors of suppliers: service level of medical institutions, medical environment for medical institutions, price of medical services, number of medical personnel, income of medical institutions, number of family doctors signing, and sinking of medical resources, etc.

(3) External factors: Jiangsu's economic development level, health policy, and medical insurance level in Jiangsu Province, etc.

\subsection{Construction of logical model of graded diagnosis and treatment system}

Through the determination of the main influencing factors and the system boundary of the grading diagnosis and treatment in Jiangsu Province. Based on these conditions this research analyzed the interaction path of each factor, and constructed the logical model of the grading diagnosis and treatment system.

Figure 2 Causal relationship diagram of grading diagnosis and treatment system in the context of medical alliances

It can be seen from Fig. 2 that the core factors for evaluating the implementation effect of graded diagnosis and treatment are affected by the recognition of the primary medical institutions and patients' willingness to grade. The realization of the first diagnosis at the primary medical institutions can promote the utilization of primary health services, and then promote the improvement of the service capacity of primary medical institutions, and the improvement of the service capacity of primary medical institutions can further enhance the patient's recognition of primary medical institutions and the willingness of 
patients to grade and treat. Form two positive feedback loops: "the first diagnosis at the primary medical institutions--primary health services utilization--primary medical institutions' service capacity-patients' willingness to grade--the first diagnosis at the primary medical institutions" and the "the first diagnosis at the primary medical institutions--primary health services utilization--primary medical institutions' service capacity--patient's recognition of primary medical institutions--patient's willingness to grade--the first diagnosis at the primary medical institutions."

The service capacity of primary medical institutions and patients' willingness to grade are the key factors in the implementation of graded diagnosis and treatment, which determines whether the graded diagnosis and treatment system can be smoothly implemented. Among them, the number of family doctors signing, primary medical institutions facilities, grassroots doctors' service level, primary health service utilization, and payability can affect the service capacity of primary medical institutions, some of the far-end factors (distant from the mediator variable) such as the grassroots service price and medical insurance compensation quota affect the service capacity of the primary medical institutions through the hierarchical transmission effect of the near-end factor (close to the median variable) such as affordability. The patient's willingness to grade is affected by factors such as the patient's physiological condition, accessibility of primary medical institutions, unreasonable medical concept, patient's income level, medical insurance differentiation level, patient's recognition of primary medical institutions, and service capacity of primary medical institutions.

Government financial compensation has increased the income of primary medical institutions, improved the facilities of primary medical institutions, raised the salary level of doctors in primary medical institutions, stimulated the enthusiasm of grassroots doctors, and improved the level of service technology of grassroots doctors to some extent. So that drive the improvement of service capabilities.

\subsection{Construction of SD model of graded diagnosis and treatment system}

\section{(1) Modeling assumptions}

Based on the system dynamics approach, in order to ensure the smooth progress of the research, the following assumptions need to be made:

Hypothesis 1:The structure of the grading diagnosis and treatment system in the context of medical alliances has relative stability. By adjusting the key factors, the problems in the diagnosis and treatment system can be alleviated to a certain extent, and then the evolution and optimization of the system structure are promoted.

Hypothesis 2: The demand side subsystem can be adjusted through a variety of factors affecting medical treatment, and factors such as medical insurance quota, health policy, population, and economy in the external environment can also influence the patient's medical needs through the path. 
Hypothesis 3: The key factors affecting the grading diagnosis and treatment system in the context of the medical alliances can be adjusted to a certain level.

\section{(2) Establish a system of flow position and flow rate variables}

In this study, the main variables of Jiangsu Province's grading diagnosis and treatment system were selected to construct the SD model『specifically as shown in Table 1. The main stock variables were the demand of hospitals and primary medical institutions. The main inflow variables were the increase in the number of visits and the amount of referrals. The main outflow variable was the number of transferred , cured, dead and lost patients.

Table 1 Variables in the graded diagnosis model

(3) Establish a flow chart of the graded diagnosis and treatment system

According to the established graded diagnosis and treatment causal relationship diagram and the constructed variable system, the flow chart of the graded diagnosis and treatment system of hospitals and prime medical institutions is established, as shown in Figure 3.

Figure 3 Flow chart of grading diagnosis and treatment system in the context of medical alliances

\section{(4) Establish mathematical function relationships}

Determine the functional relationship between the variables in the flow chart of the graded diagnosis and treatment system in the context of the medical alliances. The main function relationships involved are shown in Table 2.

Table 2 Main function relationships between variables

(5) System inspection

In the vensim software, enter the number of first diagnosis in hospitals and number of first diagnosis in primary medical institutions in Jiangsu Province in 2012-2017, then make the authentic test to verify the authenticity of the system. The results obtained are shown in Table 3.

Table 3 Simulation of the first visits of hospitals and the first visits of primary medical institutions from 2013 to 2017

It can be seen from Table 3 that except the number of first diagnosis in hospital in 2013 is $12.01 \%$, slightly higher than $10 \%$, the error rate of simulation results and the remaining numerical simulation error rates are all controlled within $10 \%$, so the model is effective and has certain Scientific basis.

\section{System Simulation}


The time period of the dynamic simulation selected in this study is 2018-2022, and the model step size is 1 year. The grassroots technical level, grassroots service price, medical insurance differentiation level, and the three indicators reflecting the construction of the medical alliances: medical resources sinking, information platform construction between medical alliance, and referral green channels of medical alliances are selected as intervention targets, and through the intervention of different targets, this study carried out systematic simulation to learn the changes in the number of first diagnosis in primary medical institutions, and then investigate the degree of impact on the implementation of graded diagnosis and treatment.

\subsection{Primary level medical technology level intervention}

The technical level is one of the explicit indicators that can directly reflect the ability of primary medical institutions. It is also a key factor affecting the patient's willingness to grade. The key to improving grassroots technical level is the reserve of grassroots health personnel, through the sinking of highquality medical resources, grassroots health personnel's skills training and reasonable salary incentives or other methods may improve the technical level of primary medical institutions. This study used it as an intervention target, and assumed that the technical level was increased by $10 \%$ on the original basis of Jiangsu Province. The results after intervention are shown in Figure 4.

Figure 4 Grassroots technical level intervention

It can be seen from Figure 4 that after raising primary medical institutions' technical level, the number of first diagnosis in primary medical institutions of Jiangsu Province has increased significantly compared with the state of non-intervention, and it has been increasing steadily during the period of 2018-2022. By 2022, the number of first diagnosis in primary medical institutions reached 41.82 million, 15.367 million higher than the state of non-intervention. It shows that the improvement of the level of primary medical technology has a positive effect on the implementation of graded diagnosis and treatment in Jiangsu Province.

\subsection{Grassroots service price intervention}

The reduction in the price level of medical services can effectively reduce the financial burden of patients and improve patient welfare. In 2017, the Jiangsu Provincial Health and Health Committee and the Jiangsu Provincial Department of Finance jointly issued the "Implementation Opinions on Promoting the Reform of Medical Service Price", clearly requiring the reasonable adjustment of the service price of primary medical institutions, and fully considering the medical insurance fund and patients' affordability, so as to construct a price system that is conducive to grading diagnosis and treatment. It shows that the adjustment of the grassroots service price is of great significance to the implementation of the grading 
diagnosis and treatment system. So that the grassroots service price is taken as the target of intervention. Assuming that the price of grassroots service is reduced by $10 \%$ on the existing basis in Jiangsu Province, the system is simulated and the result of Figure 5 is obtained.

Figure 5 Primary medical service price intervention

As can be seen from Figure 5, by reducing the grassroots service price by $10 \%$, the number of first diagnosis in primary medical institutions of Jiangsu Province has been improved to some extent, and reached 40,415,700 in 2022, compared with the non-intervention state, it has increased by 6.093 million people, so it has a significant impact on the implementation of graded diagnosis and treatment.

\subsection{Medical insurance differentiation level intervention}

By improving the differentiated payment policies for medical insurance at different levels of medical institutions, and guiding patients to orderly and rationally seek medical treatment, it is of great significance for the smooth implementation of graded diagnosis and treatment. Therefore, taking medical insurance differentiation level as the target of intervention, the supposed improvement of the level of medical insurance differentiation in Jiangsu Province is increased by $10 \%$. The simulation results of 2018-2022 are shown in Figure 6.

Figure 6 Medicare differentiation level intervention

Looking at Figure 6, we can see that by expanding the level of medical insurance differentiation, the number of first diagnosis in primary medical institutions has been significantly improved compared with the state of non-intervention. By 2022, the difference between the number in Jiangsu Province was 1169.5 , and the intervention effect was obvious.

\subsection{Medical alliances mode interventions}

In the process of promoting the graded diagnosis and treatment system in Jiangsu Province, the medical alliances mode is the main form of integration of medical resources. The purpose is to meet the different medical service needs of patients in the province by integrating medical and health services. The development of the medical alliance mode has played a powerful role in promoting the service capacity of primary medical institutions and promoting the two-way referral. Therefore, the intervention simulation of the medical alliances mode has important practical significance. This study selected three representative indicators, namely, medical resources sinking, information platform constructions between the medical alliances, and the green referral channel between medical alliances to research the influences of the implementation of the medical alliances model will bring about the promotion of the graded diagnosis and treatment system. 


\subsubsection{Increase the proportion of medical resources sinking}

The sinking of medical resources is a distinctive feature in the operation of the medical alliances mode, which is of great significance to the implementation of the grading diagnosis and treatment in the context of the current medical alliances of Jiangsu Province. The flow of high-quality medical resources from higher-level medical institutions in the medical alliances to the grassroots can promote the improvement of the health service capacity of primary medical institutions, which is conducive to improving the enthusiasm of patients' graded diagnosis and treatment. In this paper, the sinking of medical resources is selected as the intervention target, and the assumed number of medical resources sinking in Jiangsu Province is increased by $10 \%$ on the existing basis, and the simulation results shown in Figure 7 are obtained.

Figure 7 Medical resource sinking intervention

By intervening in the indicator of subsidence of medical resources, it can be found that the number of first diagnosis in primary medical institutions in Jiangsu Province after intervention in 2018-2022 has increased significantly compared with that before intervention. the number of first diagnosis in primary medical institutions in 2022 increased by 2072.9. Ten thousand people.

\subsubsection{Improve the proportion of information platform construction between medical alliances}

The integration of medical services in medical alliances mainly depends on the integration of resources, and as an auxiliary means of medical alliances construction, informatization is the best way to promote the integration and information sharing of medical alliances. Taking the information platform construction between medical alliances as an intervention target, the proportion of the medical information platform construction is supposed to be increased by $10 \%$, and the simulation results are as follows (Figure 8):

Figure 8 information platform construction between medical alliances intervention

As is shown in Figure 8, the intervention in the construction of the medical information platform can also be greatly improved. During the period from 2018 to 2022, the number of first diagnosis in primary medical institutions after intervention increased by $3.75 \%-4.20 \%$ than the status without intervention.

\subsubsection{Increase the proportion of green referral channels between medical alliances}


The two-way referral green channels are opened within the scope of the medical alliances, and the primary medical units are classified and disposed according to the patient's condition. Patients in need can be transferred to the superior hospital preferentially, so as to realize the "primary diagnosis, two-way referral, rapid division and treatment, and up and down linkage" in the true sense. The number of green referral channels between medical institutions was increased by $10 \%$ on the original basis, and the simulation results are shown in Figure 9:

Figure 9 Intervention of green referral channel between medical unions

By increasing the proportion of green referral channels between medical alliances, it can be observed that the number of first diagnosis in prime medical institutions in Jiangsu Province has increased by $3.13 \%-3.77 \%$ between 2018 and 2020, which has a significant effect on the implementation of graded diagnosis and treatment.

\subsection{Comprehensive comparison of intervention targets}

Through the above research, it is found that the same proportion of interventions for the six intervention targets can increase the number of first diagnosis in prime medical institutions in Jiangsu Province to a certain extent, which have certain roles in promoting the effect of graded diagnosis and treatment, but the level of effect of each intervention target has not yet been clarified, so this study compared the effect of each intervention target, so that the degree of effect is more intuitive. See Figure 10.

Figure 10 Comprehensive comparison of intervention targets

According to the analysis of Figure 10, the six intervention targets affecting the number of first diagnosis in primary medical institutions range from high to low level are medical resource sinking, information platform construction between medical alliances, grassroots technical level, green referral channels between medical alliances, medical insurance differentiation level, and grassroots service price. Among them, the three indicators representing the construction of the medical alliances are ranked first, second, and fourth, respectively, and the third is grassroots technical level.

\section{Conclusion}

Based on the analysis of the structural evolution of the grading diagnosis and treatment system in the context of the medical alliances in Jiangsu Province, this study grasps the dynamic mechanism of its development and changes, predicts the development of the grading diagnosis and treatment system, and simulates through relevant intervention targets to analysis the impact on the implementation of graded diagnosis and treatment. According to the modeling methods and steps of system dynamics, the system causal relationship diagram and flow graph are constructed, and the validity of the SD model is tested. The results show that the model can well reflect the internal behavior and development trend of the 
system, so it can be used for the development prediction and target interventions of the grading diagnosis and treatment system in the context of the Jiangsu medical alliances. Through the simulation study of the system, the following conclusions are obtained:

\section{(1) Target interventions have significant effects on the implementation of graded diagnosis and} treatment.

By changing the grassroots technical level, grassroots service price, medical insurance differentiation level, medical resources sinking, information platform construction and the green channels of referral between medical alliances, the number of first diagnosis in primary medical institutions has increased in varying degrees than that of non-intervention, indicating that the interventions have a positive effect on the graded diagnosis and treatment system.

(2) The construction of medical alliances is an important driving force for the successful implementation of grading diagnosis and treatment in Jiangsu Province.

By comparing the effects of each intervention target on the number of first diagnosis in primary medical institutions, it can be found that the top three impacts on the number of first diagnosis in primary medical institutions after intervention are medical resources sinking, information platform construction between medical alliances, and the grassroots technical level. Among them, the medical resources sinking and information platform construction between medical alliances who ranked in the top two are indispensable indicators for the construction of medical alliances, and the sinking of high quality medical resources in the medical alliances, and the information platform construction of the medical alliances are important means to improve the grassroots technical level. From this point of view, during the implementation of the entire grading diagnosis and treatment in Jiangsu Province, the medical alliances is the core grasper, which can promote the formation of a new pattern of medical treatment for grading diagnosis and treatment of "minor ailment treated at the grassroots level, severe illnesses treated in hospitals, and rehabilitation at the grassroots level".

\section{Discussion}

The medical alliance is an important force in the implementation of graded diagnosis and treatment. As a province that started the construction of medical alliances earlier, the construction of medical alliances have brought good effects to the implementation of grading diagnosis and treatment in the province. In order to further promote the orderly construction and development of the medical alliances of Jiangsu Province, so as to better play its role in the construction of graded diagnosis and treatment, in March 2019, the Jiangsu Provincial Health and Health Commission formulated the "Jiangsu Province Medical Complex Construction Plan (Trial Implementation )". The "Planning" clarifies that by 2020, a medical service system with reasonable layout, standardized management and high efficiency will be formed. The construction of medical alliances is gradually maturing, but there are still some problems that cannot be ignored in the process of construction. It is not conducive to the smooth implementation of graded diagnosis and treatment, and it is urgent for the relevant subjects to solve. Therefore, this study focuses 
on the following aspects to improve the implementation effect of graded diagnosis and treatment in Jiangsu Province.

(1) The medical resources sinking between the medical alliances is floating in the form, and it is necessary to fundamentally solve the problem of sinking high-quality medical resources and effectively improve the service technical level of primary medical institutions.

At present, the medical resources sinking work of the Jiangsu medical alliances is generally carried out through a single form of experts from tertiary hospitals providing medical services and regular ward rounds. Only when the experts are in clinic, the learning of grassroots medical technology can be carried out. Once the experts withdraw, the technology learning is also stagnated, so the support for primary medical institutions is still superficial, and haven't prove a substantive solution to the problem. From the perspective of the demand side, when the patient chooses their first medical institution, the starting point is that the disease can be cured or improved at once. The rehabilitation rate of the first diagnosis can have an important impact on the Primary medical institutions first diagnose ratio ${ }^{[14]}$. Therefore, it is the key to graded diagnosis and treatment to improve the level of service technology of primary medical institutions. The members of the medical alliances are cooperative relationship. For tertiary hospitals in the medical alliances, the management of the member institutions, the quality control, personnel training, technical assistance and other resources sinking tasks should not only be implemented, but also to take root. Therefore, on the one hand, it is necessary to encourage the establishment of joint wards, disciplinary centers, and family medicine alliances in the medical alliances, to implement implantable assistance for technologies developed in the primary medical institutions, implement homogenization management, and strengthen the construction of grassroots discipline level. On the other hand, with the establishment of the "Internet + medical health" service system, the medical alliances should accelerate the promotion of telemedicine, assist the grassroots doctors to better carry out their work, and promote the orderly grading diagnosis and treatment pattern of "examine at the grassroots level, diagnose at superior institutions ". Implement the sinking of high-quality resources and fundamentally improve the service technical level of primary medical institutions.

(2) The information technology standards in the medical alliances have not been unified, and there still exist information islands. It is necessary to strengthen the construction of information platform and promote the smooth implementation of two-way referral.

Information technology is the cornerstone of the medical alliances, and it also penetrates into all aspects of the medical alliances. Although the construction of the medical alliances in Jiangsu Province has achieved certain results so far. However, in the medical alliances, the patient information is actually obstructed. The health record management system between different institutions is not uniform and incompatible. The medical records of the referral patients cannot be shared. The information of each medical institution is only publicized internally, and the information islands are widespread. This causes the information on the implementation of the graded diagnosis and treatment to be incoordinate and other possible issues. Therefore, in order to solve the above-mentioned situation, to achieve 
interconnection and mutual enjoyment between the medical alliances, we should use the "Internet + health" technology as a support, accelerate the pace of information construction, strengthen the integration of information resources, then guide and supervise the members of the medical alliances to standardize all electronic files. Under the premise of ensuring information security, the institutions in medical alliances can moderately share data, open up information barriers, establish a comprehensive and unified health information platform, then connect the isolated information systems of hospitals to realize multi-directional transmission and real-time deployment of doctor-patient data, so as to provide support for the smooth transfer of two-way referrals.

(3) Broaden the green channels between the medical alliances and optimize the two-way referral process.

The construction of the two-way referral green channels in the medical alliances is a bridge of clinical specialties between the primary medical institutions and the tertiary hospitals, which is conducive to standardizing the referral evaluation criteria and referral routes, and truly realizing the two-way referral mode with the integration of the tertiary hospitals - primary medical institutions. There is still lack of work in the construction of green referral channels in Jiangsu Province. Therefore, it is necessary to pay attention to widening the two-way referral green channels between the medical alliances and continuously optimizing the two-way referral process. In the construction of the medical alliances, the community management department can be set up in the tertiary hospitals to maintain close contact with the primary medical institutions in the medical alliances, specifically responsible for the support work of the community hospitals and patient referral work, providing green channels for two-way referrals, so that patients who face the need for referral can obtain the priority to the referral, and optimize their referral processes. At the same time, patients who sign up in the medical alliances can get proper medical technical support, and a reasonable follow-up rehabilitation program to improve their willingness to grade, ensure the smooth implementation of the two-way referral, and realize the mode of "minor ailment treated at the grassroots level, severe illnesses treated in hospitals, and rehabilitation at the grassroots level".

(4) Exploring the medical insurance reform in the medical alliances, implementing the differentiation of medical insurance policies, and accelerating the establishment of a linkage mechanism for medicaltreatment, drug and insurance.

At this stage, the deep-seated reason why the medical alliances are not linked in the body is that the linkage mechanisms of medical treatment, drug, and medical insurance work have not yet been established. The medical insurance reimbursement and financial subsidies in the medical alliances are lagging behind, and patients' awareness of graded diagnosis and treatment is weak. Therefore, in order to better implement the grading diagnosis and treatment within the medical alliances, the medical unit should be assessed as a whole to exert the leverage of medical insurance, open the medical insurance payment channel, ensure the homogenization of medicines at all levels of medical institutions, and prevent patients who go down to the primary medical institutions cannot reach the required drugs. Secondly, it is necessary to build a basic medical security system that is compatible with economic 
development, improve the multi-level security system, focus on promoting the reform of medical insurance payment methods based on disease-based payment, and expand the registration of some medical treatments at various levels of medical institutions in the medical alliances. The gap between the fee, the price of medical treatment and the proportion of reimbursement depends on the differentiated payment incentives to enable patients to form scientific consumption habits, gradient consumption behaviors, and gradually guide patients to diagnose in primary medical institutions. Finally, combined with the grading diagnosis and treatment mode and the establishment of the family doctor sign service system, the insured patients in the medical alliances who meet the referral requirements are continuously calculated their staring pay line, and the eligible family doctors' contract service fees are included in the scope of medical insurance payment, to reasonably guide the two-way referral, and play the "gatekeeper" role of family doctors in medical insurance control fees.

(5) Improve the mechanism of sharing benefits among medical alliances, establish common points of benefits, and put an end to the phenomenon of "siphoning" in hospitals.

The medical alliances' referrals in the process of promoting the implementation of graded diagnosis and treatment lead to the flow of patients. For core hospitals, the transfer of patients means the loss of benefits, so patients who are "profitable" are usually kept in the hospital, and patients who are "unprofitable" are transferred to the grassroots, lacking the real motivation to turn patients down, so that may cause the hospital's "siphon" phenomenon. The primary medical institutions in the medical alliances think that the economic benefits generated by the patients under the transfer are far less than those of the first diagnose patients. Therefore, they are reluctant to receive rehabilitation patients whose benefits are not obvious in the later period. There is a divergence of interests among the members of the medical alliances. The relationship between medical alliances members has evolves into a competitive one, which is contrary to the original intention of two-way referral of graded diagnosis and treatment, and has become the most essential reason for obstructing graded diagnosis and treatment. Therefore, the primary task of the construction of the medical alliances is to improve the mechanism of benefit sharing among the members of the medical alliances, to reach an agreement on the distribution of benefits between the core hospitals and member hospitals, and to innovate personnel protection and compensation incentives, abolish performance indicators, and turn to fully mobilizing the enthusiasm of medical staff by material and spiritual incentives for two-way referral. At the same time, a clear two-way referral mechanism should be established within the medical alliances, and the unified referral clinical standards, management norms and evaluation criteria should be published in the form of official documents, to realize the patient's "severe illness be referred upwards, rehabilitation be referred downward" ,to promote the effective diagnosis and treatment policy, and effectively prevent the phenomenon of "siphon".

\section{Declarations}

\section{Data Avalibility}




\section{Conflicts of Interest}

THE AUTHOR DECLARES THAT THEY HAVE NO CONFLICTS OF INTEREST.

\section{Acknowledgments}

THIS PAPER IS SUPPORTED BY KEY PROJECT OF PHILOSOPHY AND SOCIAL SCIENCE RESEARCH IN JIANGSU UNIVERSITY, N0.2018SJZDI074, GRADED DIAGNOSIS AND TREATMENT SYSTEM AND POLICY SIMULATION RESEARCH BASED ON SD MODEL IN THE BACKGROUND OF JIANGSU MEDICAL ALLIANCES CONSTRUCTION.

Fund Project: Key Project of Philosophy and Social Science Research in Jiangsu University, 2018SJZDI074, Graded Diagnosis and Treatment System and Policy Simulation Research Based on SD Model in the Background of Jiangsu Medical Alliances Construction

Nanjing University of Chinese Medicine, Nanjing, Jiangsu 210023

Tianjing Normal University, Tianjing 300387

The first author profile: Shaoliang Tang (1969-), male, doctor, professor, doctoral tutor, research direction: health management, drug policy, etc., E-mail: tangshaoliang@126.com, Tel: 13952003436

Jiahuan Qiu(1995-), female, graduate student, research direction: health management, drug policy, etc.

\section{References}

[1] J. Lu. "On the improvement of the grading diagnosis and treatment system in the course of deepening medical improvement”. Chinese Hospital Management, vol 34, no.6, pp. 1-3,2014.

[2] S.-C. He, D.-R. Zhao, R.-H. Zhang, et al. “Implementation status and thinking of graded diagnosis and treatment in China”. Modern Hospital Management, vol 13, no.2, pp. 20-22, 2015.

[3] H.-X. Yu, W. Yao, Y. Li, et al. "Research on the division mechanism of medical institutions based on graded diagnosis and treatment". Hospital Management, vol 34, no.7, pp.1-3, 2014.

[4] N.-Y. Mao, D.-D. Ma. "Current status and optimization suggestions for the classification and treatment system in Jiangsu Province” . Modern Trade and Industry, vol 39, no. 17, pp. 23-25, 2018.

[5] Fallah-Fini S, Rahmandad H, Huang T T, et al. "Modeling US adult obesity trends: a system dynamics model for estimating energy imbalance gap”. American Journal of Public Health \vol 104, no.7, pp. 1230- 
1239, 2014.

[6] Tobias M I, Cavana R Y, Bloomfield A. "Application of a system dynamics model to inform investment in smoking cessation services in New Zealand". American Journal of Public Health $\llbracket$ vol 100, no. 7, pp. 1274-1281, 2010.

[7] FORRESTER J W『“Industrial dynamics” $₫$ Cambridge MA:Productivity Press, 1961.

[8] Y.-B. Zeng, T.-J. Yang, W. Xu, and Y. Fang. "Research on the System Dynamics of China's Medical Insurance System under the Background of Aging". Chinese Journal of Health Statistics, vol 35, no.6, pp. 811-816, 2018.

[9] X. Li. "Social System Dynamics". Shanghai: Fudan University Press, 2008.

[10] W. Zhang. "Hierarchical diagnosis and treatment system and policy simulation based on system dynamics model" . Zhejiang University, 2017.

[11] Q.-Q. LU, J.-H. Wang. "Analysis of Factors Affecting Warehouse Fire Accidents Based on System Dynamics". Journal of Safety and Environment, vol 18, no. 5, pp. 1767-1773, 2018.

[12] W. Zhang, Y. Liu, S.-Y. Gu, X.-M. Qi, Y.-H. Zeng, W. Gu, H.-J. Dong. "Whether tiered medical care system is effective in saving costs? Cost savings estimation of themedical care system after tiered optimization in Zhejiang Province based on system dynamics model". China Health Policy Research, vol 10,no. 7, pp. 13-21, 2017.

[13]Y. Zhang. "Research on Several Issues of Energy Saving and Emission Reduction Strategy of Logistics System” . Beijing Jiaotong University, 2011.

[14] X. R Li, J.-F. Tian, X.-Y. Li, C. Wang, and W. Tang. "Research on the construction of evaluation index system for the first-level implementation of primary health care based on the perspective of demand side". Chinese Journal of Pharmaceutical Economics, vol 14, no. 2, pp.5-10. 2019.

\section{Tables}

Table 1 Variables in the graded diagnosis model 


\begin{tabular}{|c|c|}
\hline $\begin{array}{l}\text { Variable } \\
\text { category }\end{array}$ & Variables \\
\hline Horizontal & Total hospital demand \\
\hline variables & Total demand of primary medical institutions \\
\hline \multirow[t]{3}{*}{ Rate variables } & Increase in hospital and primary medical institutions \\
\hline & $\begin{array}{l}\text { The amount of hospitals and primary medical institutions the number of transferred, cured, dead } \\
\text { and lost patients. }\end{array}$ \\
\hline & $\begin{array}{c}\text { The amount of hospital downward referrals, The amount of primary medical institutions upward } \\
\text { referrals }\end{array}$ \\
\hline Auxiliary & Annual visits \\
\hline variables & Increase in hospital and primary medical institutions visits \\
\hline \multirow[t]{2}{*}{ Initial variables } & Annual visits, Primary medical institutions first diagnosis ratio \\
\hline & Upward and downward referral rate between hospitals and primary medical institutions \\
\hline
\end{tabular}

Table 2 Main function relationships between variables

\begin{tabular}{|c|c|}
\hline Variables & Function relationships \\
\hline $\begin{array}{l}\text { The number of first } \\
\text { diagnosis in hospitals }\end{array}$ & Annual visits* Hospital first diagnosis ratio \\
\hline $\begin{array}{l}\text { Jumber of first diagnosis in } \\
\text { Primary medical } \\
\text { institutions }\end{array}$ & Annual visits* Primary medical institutions first diagnosis ratio \\
\hline $\begin{array}{l}\text { Number of hospitals' } \\
\text { outpatients }\end{array}$ & Number of first diagnosis in hospitals* downward referral rate \\
\hline $\begin{array}{l}\text { Jumber of primary medical } \\
\text { institutions' outpatients }\end{array}$ & Number of first diagnosis in primary medical institutions* upward referral rate \\
\hline Total hospital demand & $\begin{array}{c}\text { Number of first diagnosis in hospitals +Increase in hospital visits - number of cured, } \\
\text { dead, and lost patients in hospitals }\end{array}$ \\
\hline $\begin{array}{l}\text { Total demand of primary } \\
\text { medical institutions }\end{array}$ & $\begin{array}{c}\text { Number of first diagnosis in primary medical institutions }+ \text { increase in primary } \\
\text { medical institutions visits - number of cured, dead, and lost in primary medical } \\
\text { institutions }\end{array}$ \\
\hline
\end{tabular}

Table 3 Simulation of the first visits of hospitals and the first visits of primary medical institutions from 2013 to 2017 


\begin{tabular}{|c|c|c|c|c|c|c|}
\hline \multirow[t]{2}{*}{ Year } & \multicolumn{3}{|c|}{$\begin{array}{l}\text { Number of first diagnosis in hospitals } \\
\qquad(10,000)\end{array}$} & \multicolumn{3}{|c|}{$\begin{array}{l}\text { Number of first diagnosis in primary medical institutions } \\
\qquad(10,000)\end{array}$} \\
\hline & $\begin{array}{l}\text { Actual } \\
\text { value }\end{array}$ & Simulation result & Error rate & Actual value & Simulation result & Error rate \\
\hline 2013 & 21201 & 23746.2 & $12.01 \%$ & 27071.57 & 25674 & $-5.16 \%$ \\
\hline 2014 & 23003 & 24585.8 & $6.88 \%$ & 28455.61 & 28083.1 & $-1.31 \%$ \\
\hline 2015 & 24121.83 & 25235.1 & $4.62 \%$ & 29201.6 & 29393.1 & $0.66 \%$ \\
\hline 2016 & 24754.7 & 24650.5 & $-0.42 \%$ & 29116.3 & 30565.5 & $4.98 \%$ \\
\hline 2017 & 25717 & 24426.7 & $-5.02 \%$ & 31338 & 34010.3 & $8.53 \%$ \\
\hline
\end{tabular}

\section{Figures}

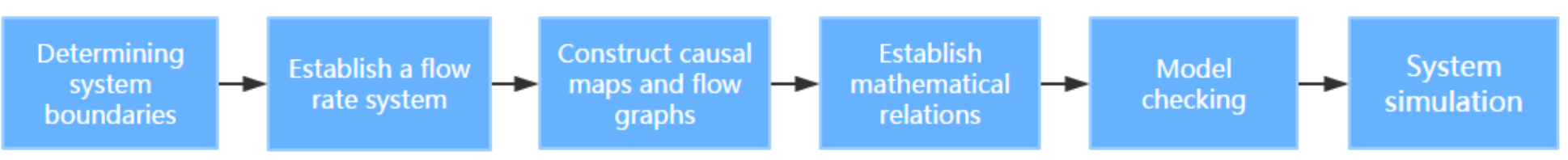

\section{Figure 1}

Grading diagnosis and treatment system dynamics modeling steps

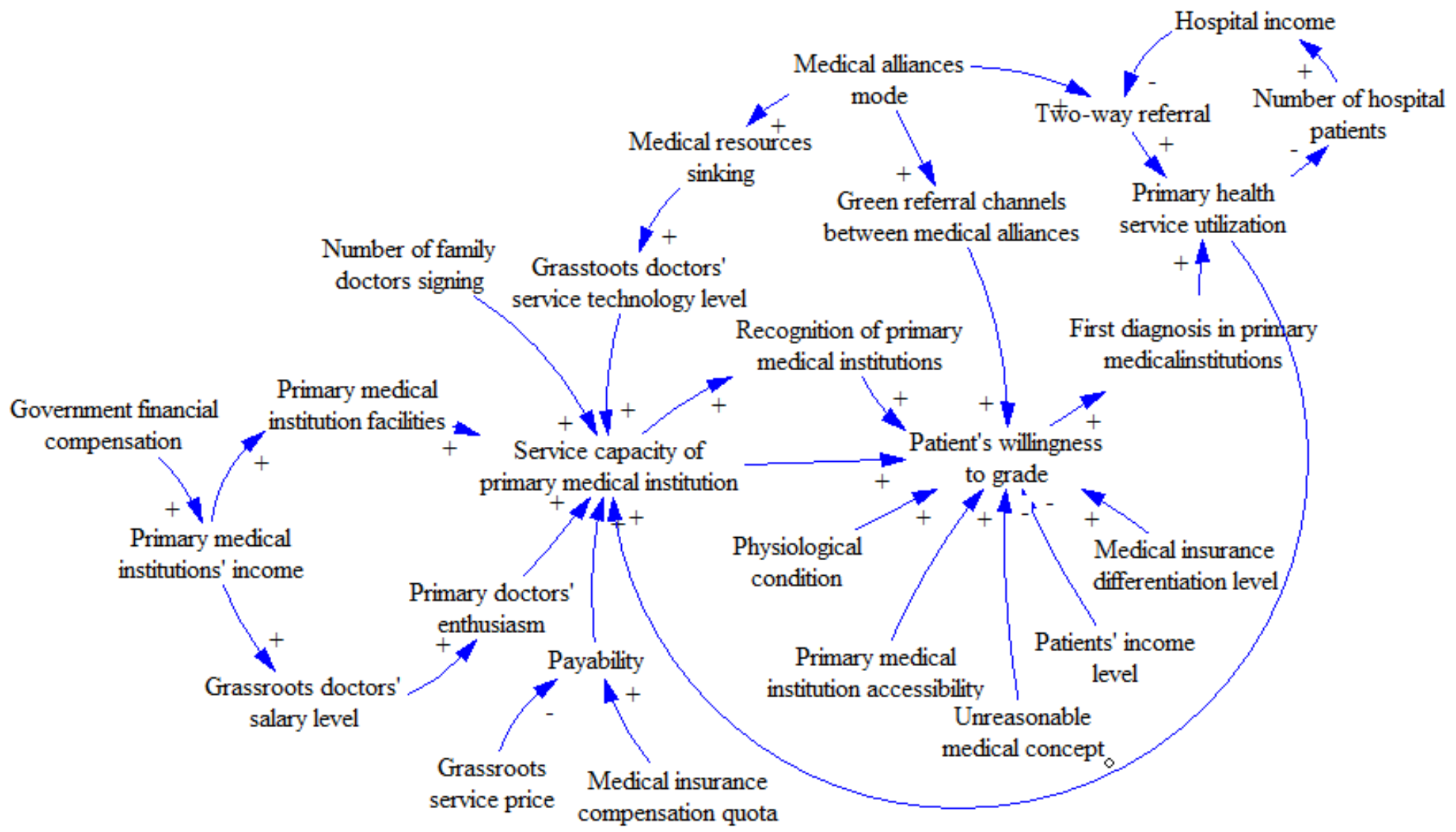


Causal relationship diagram of grading diagnosis and treatment system in the context of medical alliances

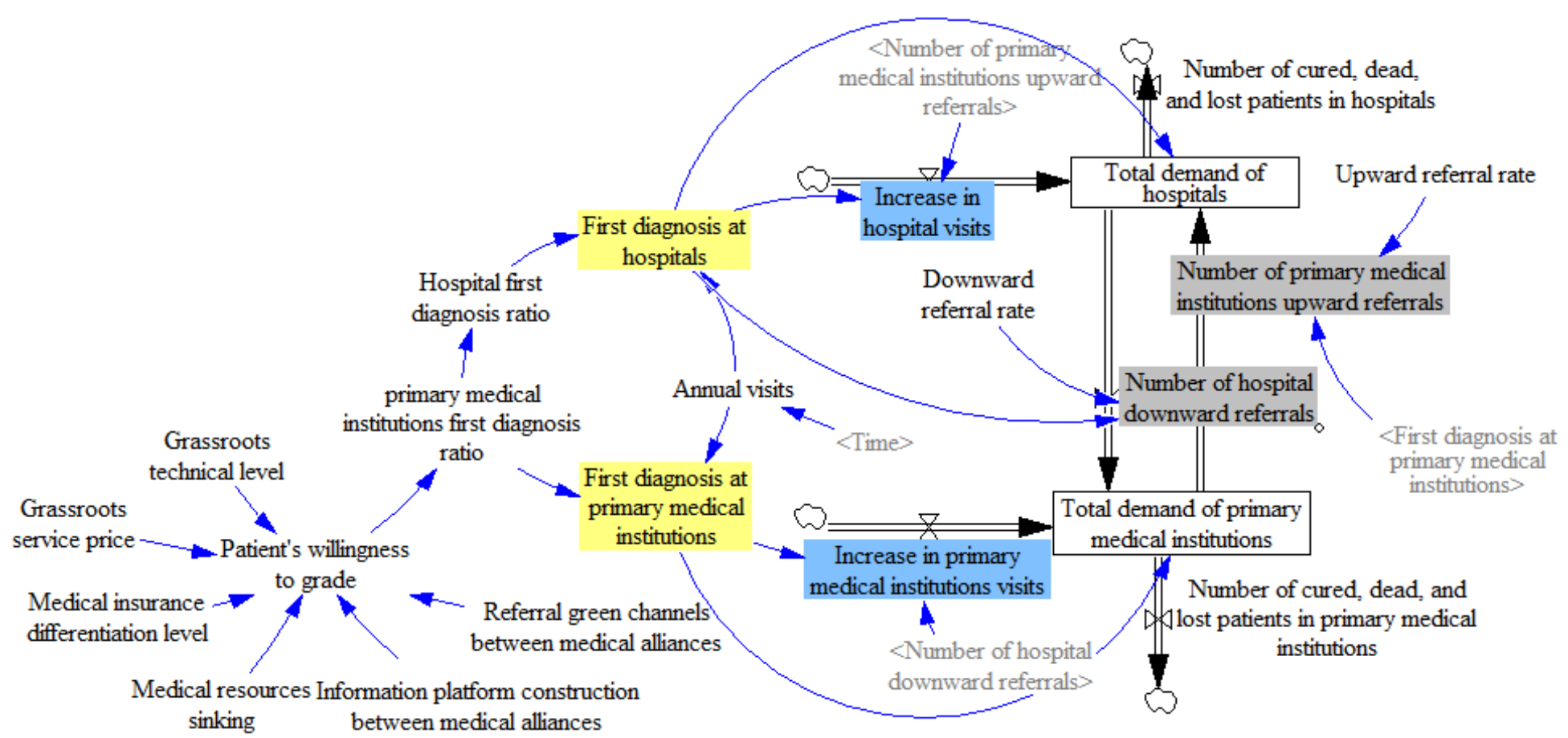

\section{Figure 3}

Flow chart of grading diagnosis and treatment system in the context of medical alliances

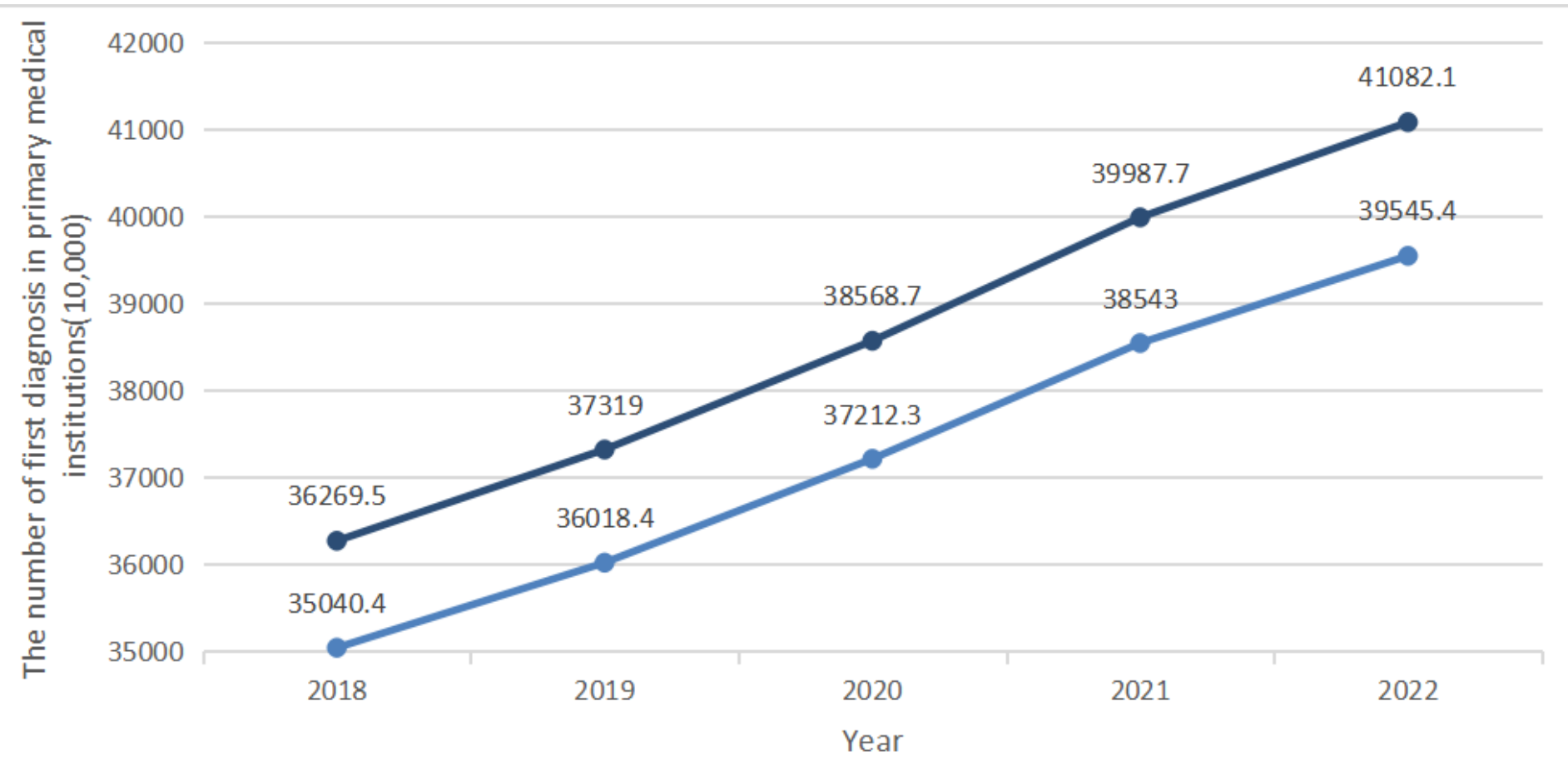

No intervention Grassroots technical level increased by $10 \%$

\section{Figure 4}


Grassroots technical level intervention

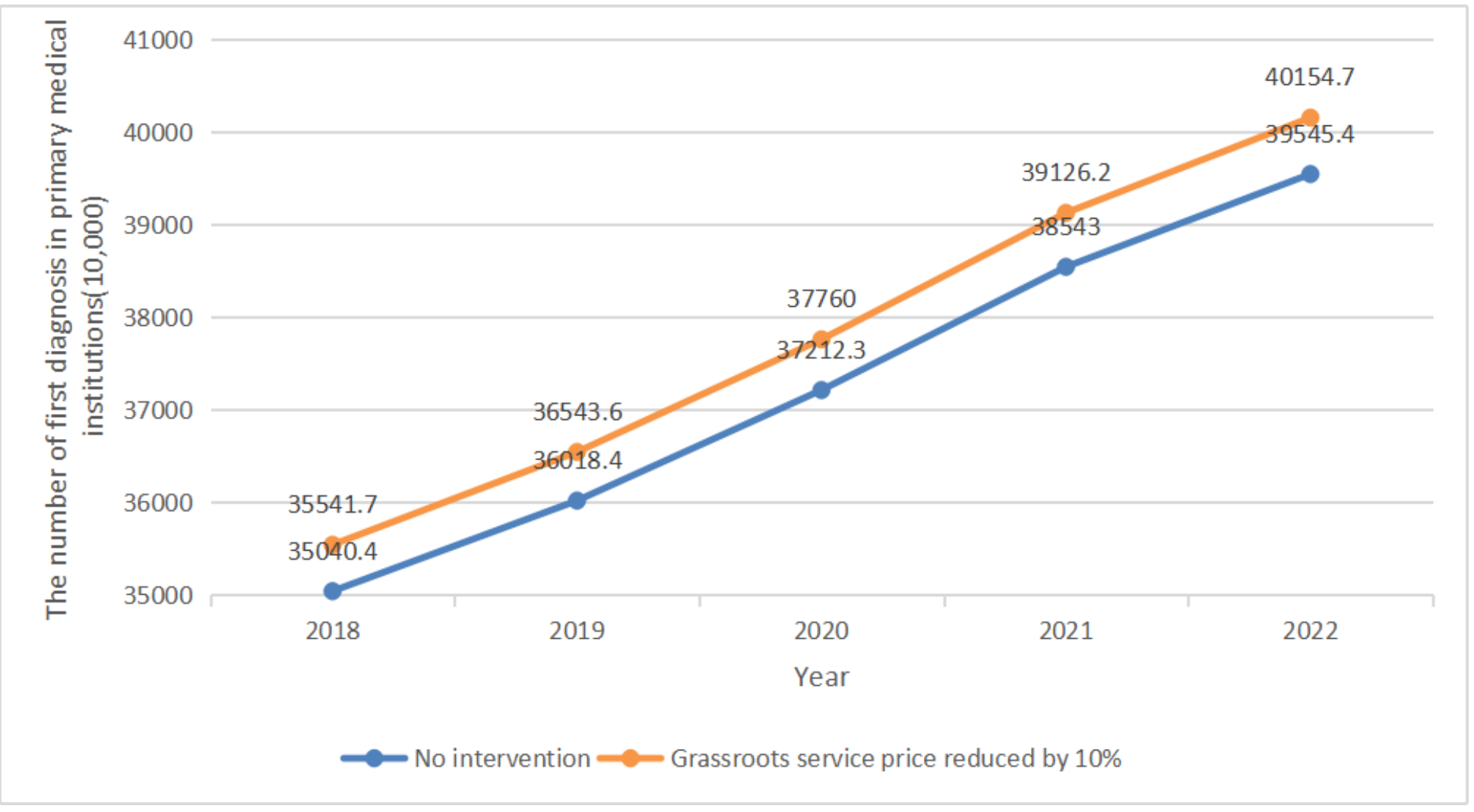

\section{Figure 5}

Primary medical service price intervention

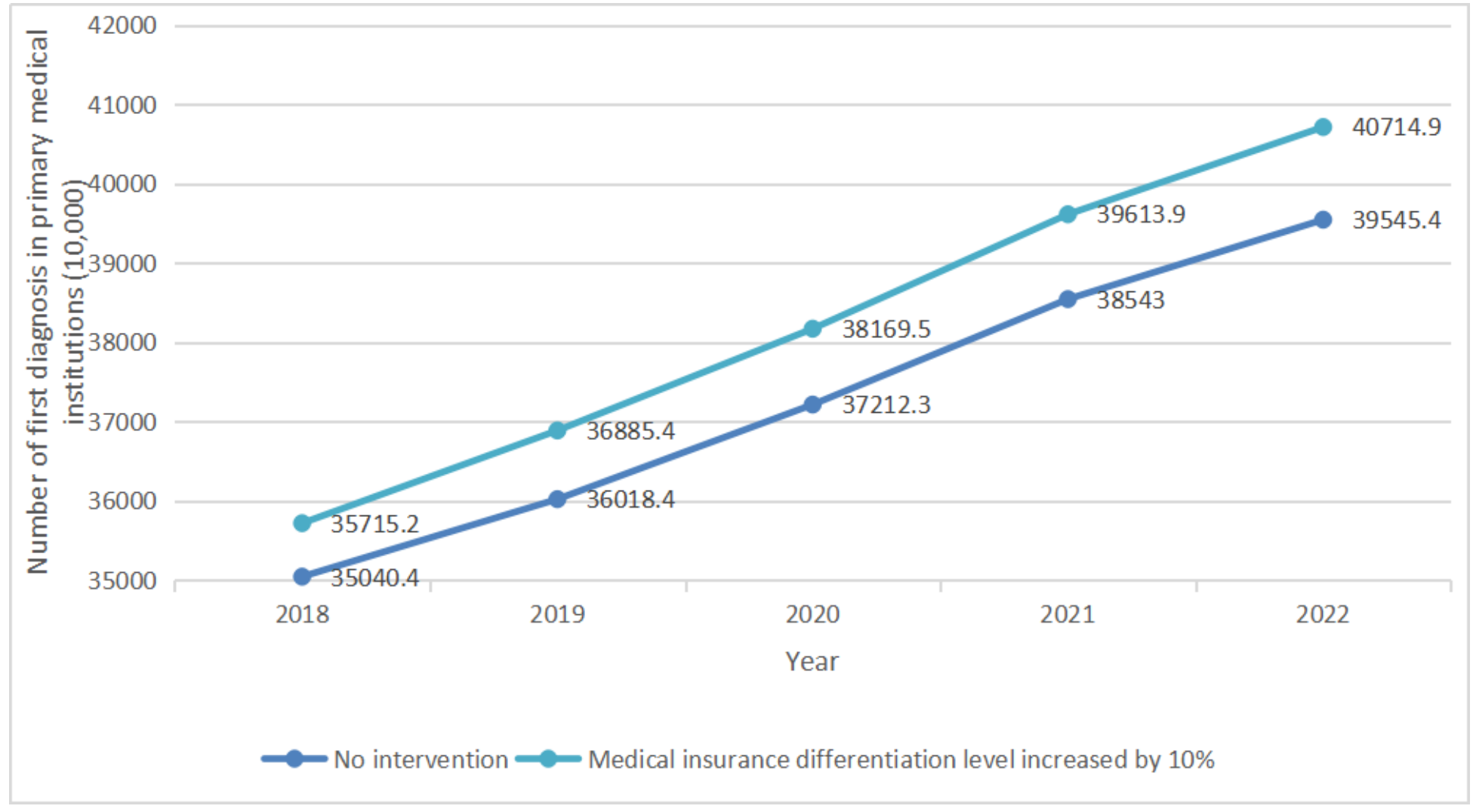

Figure 6 
Medicare differentiation level intervention

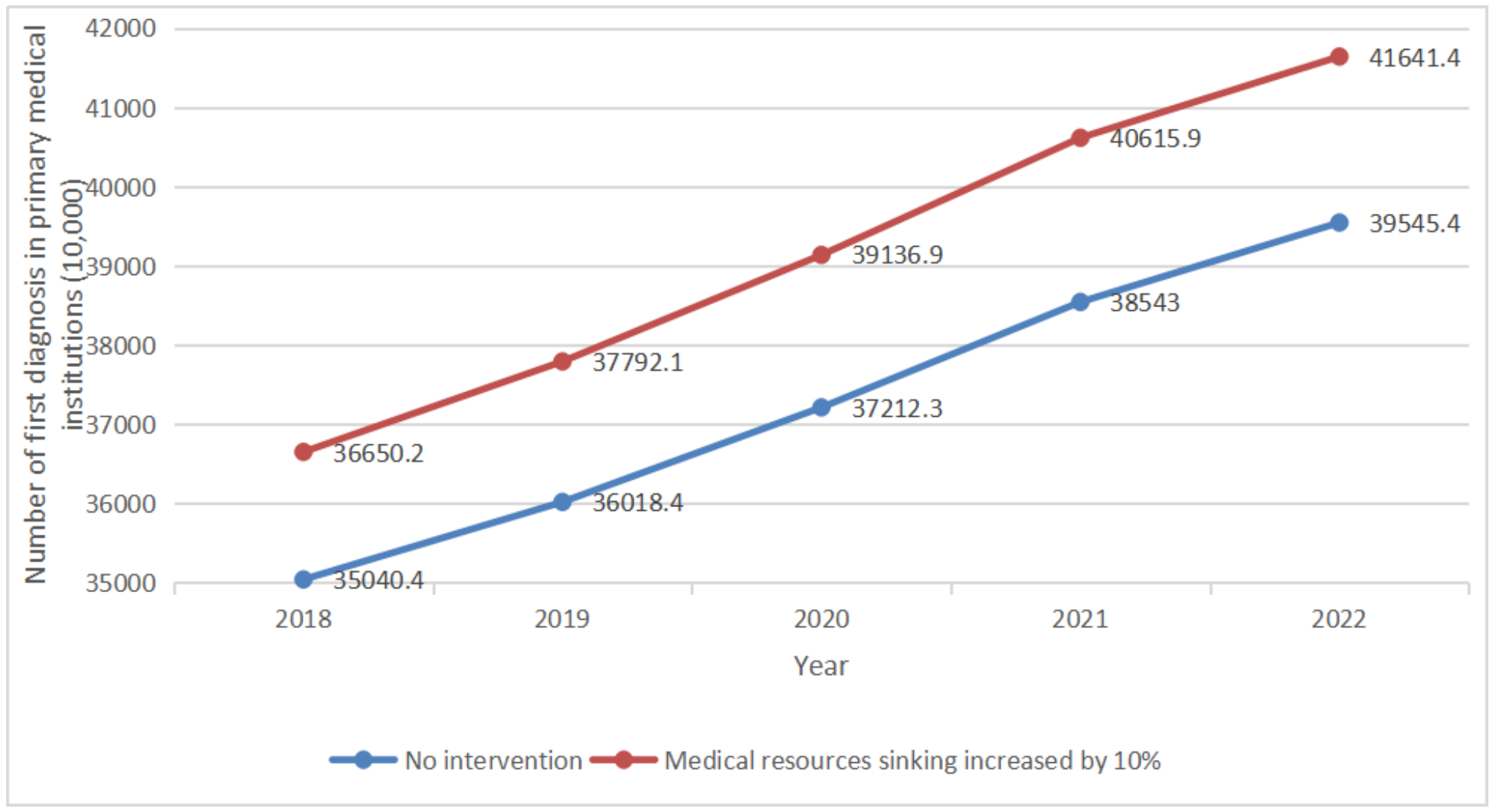

\section{Figure 7}

Medical resource sinking intervention

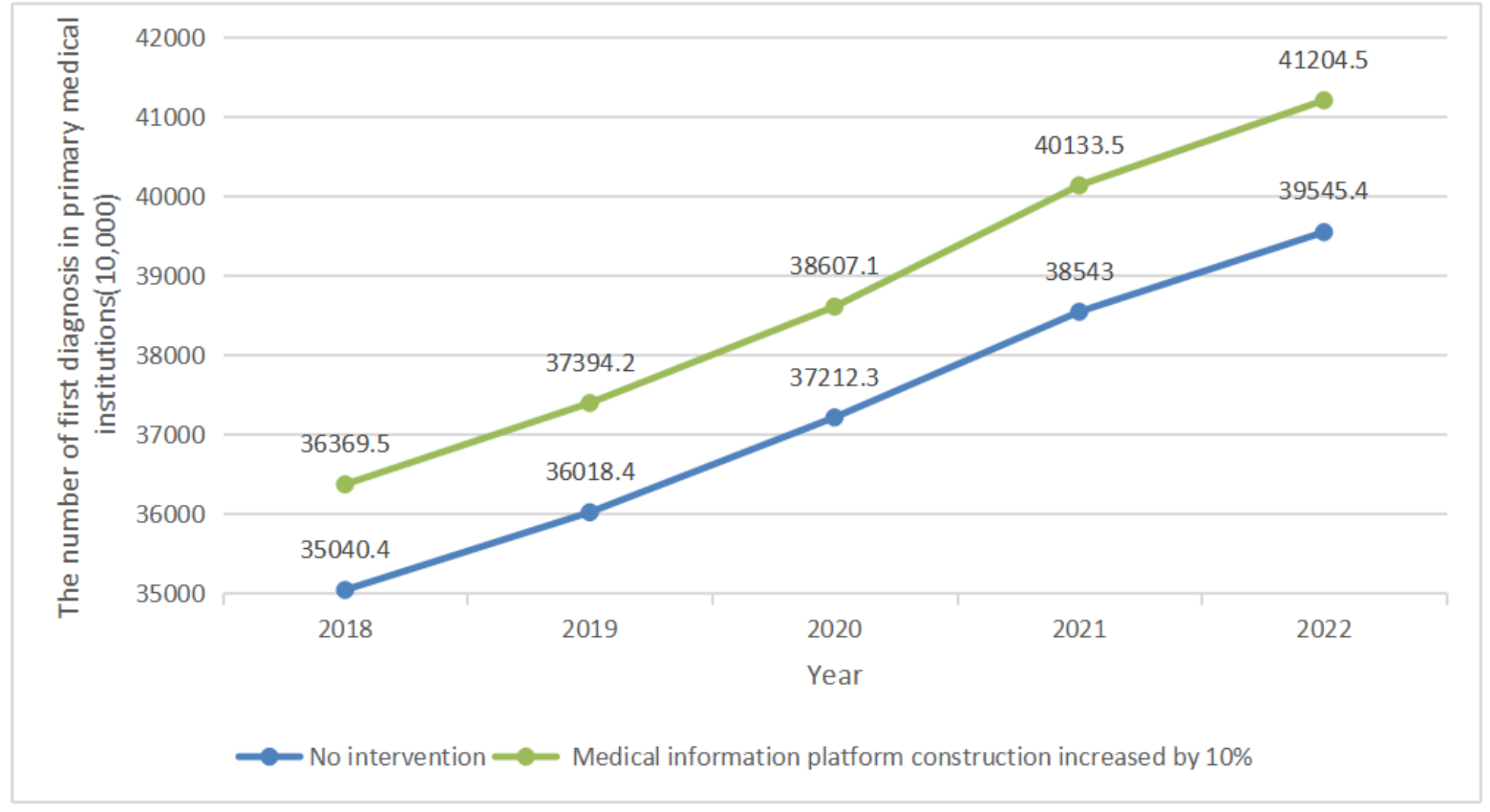

Figure 8 
information platform construction between medical alliances intervention

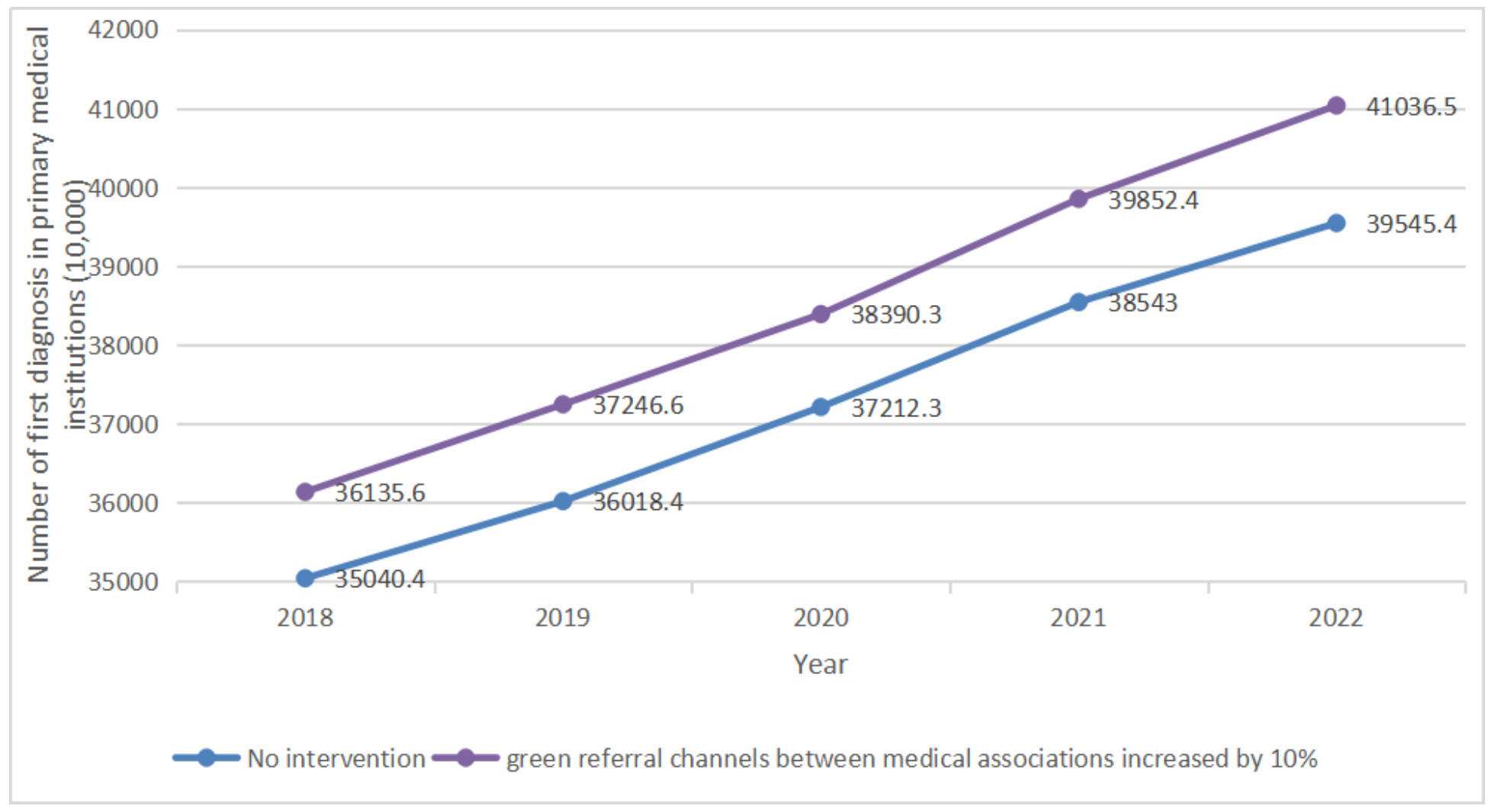

\section{Figure 9}

Intervention of green referral channel between medical unions 


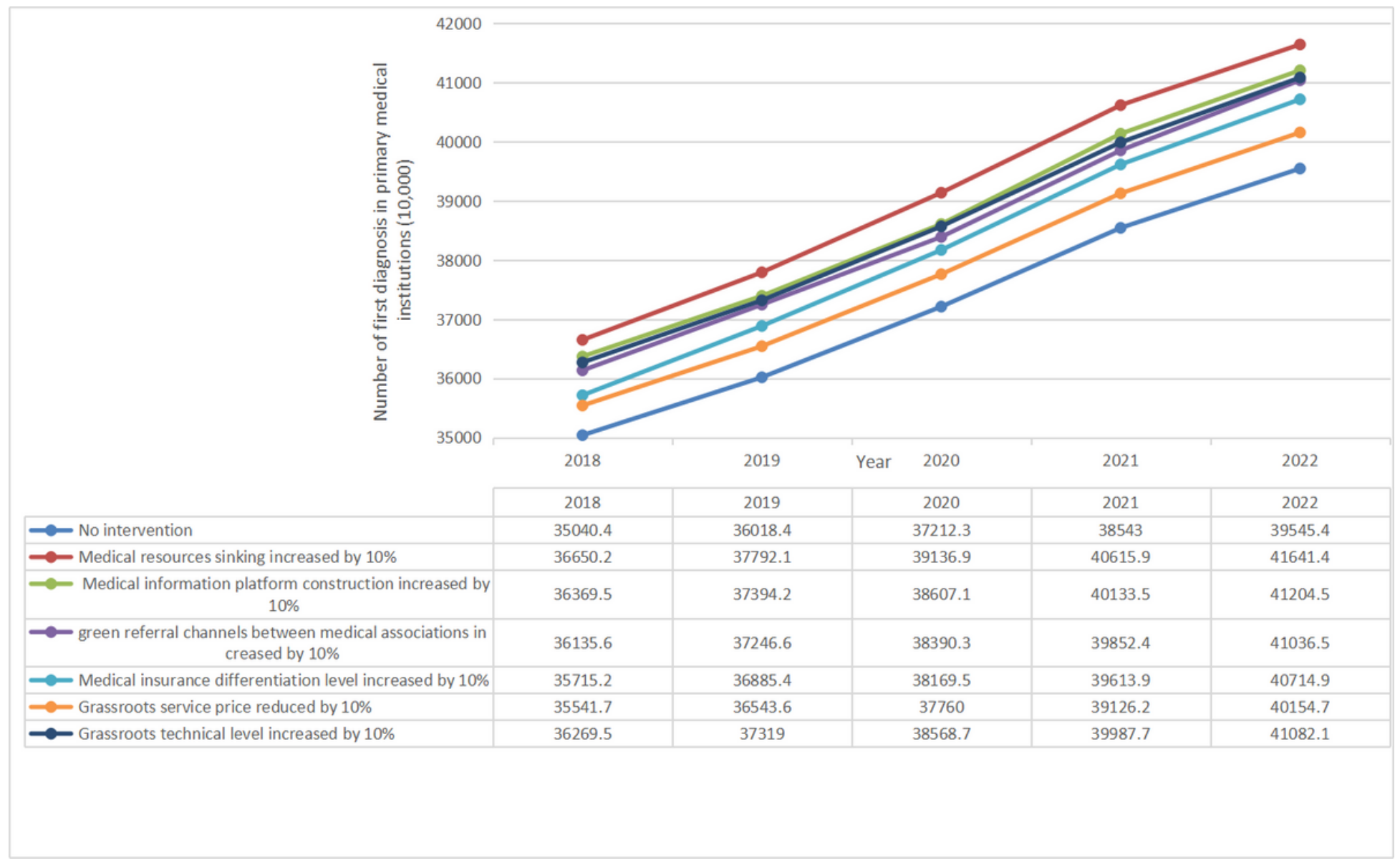

Figure 10

Comprehensive comparison of intervention targets 